\title{
Hyperon weak radiative decays in chiral perturbation theory
}

\author{
J.W. Bos ${ }^{1}$, D. Chang ${ }^{2,1}$, S.C. Lee ${ }^{1}$, Y.C. Lin, and H.H. Shih ${ }^{3}$ \\ ${ }^{1}$ Institute of Physics, Academia Sinica, Taipei, Taiwan \\ ${ }^{2}$ Department of Physics, National Tsing Hua University, Hsinchu, Taiwan \\ ${ }^{3}$ Department of Physics and Astronomy, National Central University, Chungli, Taiwan
}

We investigate the leading-order amplitudes for weak radiative decays of hyperons in chiral perturbation theory. We consistently include contributions from the next-to-leading order weak-interaction Lagrangian. It is shown that due to these terms Hara's theorem is violated. The data for the decays of charged hyperons can be easily accounted for. However, at this order in the chiral expansion, the four amplitudes for the decays of neutral hyperons satisfy relations which are in disagreement with the data. The asymmetry parameters for all the decays can not be accounted for without higherorder terms. We shortly comment on the effect of the 27-plet part of the weak interaction.

\section{INTRODUCTION}

Weak radiative decays of hyperons, $B_{i} \rightarrow$ $B_{f}+\gamma$, have received attention for a long time, both experimentally [1-6] and theoretically 7720 . There have been several theoretical approaches to this problem. One of the two major approaches uses hadronic degrees of freedom [9] 12 while the alternative is solely based on the quark picture of hyperons 13 15. While the data are known to be consistent with the lower bounds on the amplitudes derived from unitarity constraints [9, 11], none of the theoretical models have managed to give a satisfactorily account on details of the data, in particular, the rates for the four neutral decay modes.

One of the issues that has been emphasized in the literature is the apparent violation of Hara's theorem [7, 8], which states that the parity-violating amplitudes for $\Sigma^{+} \rightarrow p+\gamma$ and $\Xi^{-} \rightarrow \Sigma^{-}+\gamma$ vanish in the limit of $S U(3)$ symmetry. It predicts, in contradiction with experiments, that the asymmetry parameter for $\Sigma^{+} \rightarrow p+\gamma$ should be quite small. (See Ref. [21] for a review of the relevant arguments.)

The hadronic models did not have a great deal of success in explaining the details of the data. All the models of this type (except that which include vector mesons) pre- serve Hara's theorem in their formulations. General analysis which include $S U(3)$ breaking 13] actually predicts a small and positive asymmetry for the $\Sigma^{+}$decay while the data shows that it is negative and relatively large. Models that assume vector-meson dominance 16 can introduce effects that violate Hara's theorem due to mixing of the vector meson with the photon. In models using quarks, it was pointed out [14 that the diagrams in which a $W$-boson is exchanged between two constituent quarks can give rise to violation of Hara's theorem. In addition, models which include vectormeson dominance are in better agreement with the data, though the situation is still not satisfactory. The experimentally observed negative asymmetry parameter for $\Sigma^{+}$decay is best accounted for using QCD sum-rules [17]. Other approaches can be found in Refs. 18 20]. A detailed overview on both experimental and theoretical aspects of weak radiative decays of hyperons is given in Refs. 4, 21, 22.

Chiral

perturbation theory (ChPT) 23,24 has been shown to be a useful way of describing low energies hadronic processes, especially those that involve only mesons. It is an effective field theory in terms of hadronic degrees of freedom, based on the symmetry properties of QCD. For application to processes involving baryons, it is most consistently formulated in the heavy-baryon formulation [25], in which the $S U(3)$ invariant baryon mass, $\dot{m}$, is removed by a field transformation (see also Ref. 26], where a similar transformation is carried out). In this approach an amplitude for a given process is expanded in external pion four-momenta, $q$, baryon residual four momenta, $k$, and the quark mass, $m_{s}$. We will neglect the up and down quark mass. We will collectively write down $q, k$, and $m_{s}$ as $E$. (As we will discuss later, we will adopt the convention that $k$ and $m_{s}$ are of the same order in the chiral expansion.) The perturbation theory is reliable only when $E$ is smaller than the chiral 
symmetry breaking scale $\Lambda_{\chi}$. In the heavybaryon formulation there is an additional expansion in $1 / \dot{m}$. However, all these terms can be effectively absorbed in counterterms of the theory [27.

Weak radiative decays of hyperons have been studied before in the context of ChPT by Jenkins, Luke, Manohar and Savage [20] and Neufeld 19. Jenkins et al. and Neufeld calculated the amplitude up to the oneloop level. These loop diagrams give contributions to the amplitudes which are at least of order $O\left(E^{2}\right)$ in the chiral expansion. However, tree-level direct emission diagrams from the next-to-leading order weak Lagrangian [27], which give contribution of order $O(E)$ to the amplitudes, were not considered. The reasons such terms might be neglected consistently is the fact that they are not needed for renormalization. However, in general, since ChPT should be based on a most general Lagrangian 24, they should be included also. We will see that as a consequence of not taking these terms into account, the analysis for weak radiative decay of both Jenkins et al. and Neufeld should satisfy Hara's theorem.

In this paper we consistently calculate the leading-order amplitude of weak radiative decays of hyperons in ChPT. At this order, no loop contribution need to be considered. However, one does need to take into account the higher-order terms in the weak chiral Lagrangian. We will show that it gives rise to violation of Hara's theorem. As a consequence the decay rates for the charged decays $\Sigma^{+} \rightarrow p+\gamma$ and $\Xi^{-} \rightarrow \Sigma^{-}+\gamma$ can be accounted for consistently. We will show that, in leading order, ChPT predicts the ratios of the decay amplitudes of all the neutral channels as functions of the baryon masses only. We will compare these predictions with the data. Furthermore, the asymmetry parameters still vanish in this leading order calculation. We shall explain why this is not necessarily inconsistent with the data in the expansion scheme of ChPT. We will also discuss the contribution of the 27-plet component of the weak Lagrangian to the amplitude.

This paper is organized as follows. We will start in the next section by discussing the general formalism of hyperon weak radiative decay. In Sec. III we will calculate the amplitude in leading order ChPT, including contributions from the weak and strong inter- action Lagrangian with higher order terms. In Sec. IV we will discuss briefly the contribution from the 27-plet. Next, in Sec. V, the parameters appearing in the expressions are discussed and compared with data. Finally, Sec. VI contains a summary and our conclusions.

\section{GENERAL FORMALISM}

In this section we will consider some general features of weak radiative hyperon decay, and summarize the data. To deal with baryons, we will work in the heavy-baryon formalism 25], briefly outlined in the appendix.

As shown in the appendix, in the heavybaryon formalism and in the gauge

$$
v \cdot A=0
$$

the general amplitude for the hyperon weak radiative decay process

$$
B_{i}\left(m_{i}\right) \rightarrow B_{f}\left(m_{f}\right)+\gamma
$$

is given by

$$
\begin{aligned}
& \epsilon_{\mu}(q) \mathcal{M}^{\mu}= \\
& \quad 2 \epsilon_{\mu}(q) \bar{U}_{v}\left(k^{\prime}\right)\left(q_{\nu}\left[S_{v}^{\mu}, S_{v}^{\nu}\right] A+\Delta m S_{v}^{\mu} B\right) U_{v}(k),
\end{aligned}
$$

where $U_{v}$ and $\bar{U}_{v}$ are the heavy-baryon spinors of the initial and final baryons, respectively, and $\epsilon_{\mu}$ is the photon polarization vector. The momenta are defined in Fig. 1. The "form factors" $A$ and $B$ in Eq. (2) correspond to the parity-conserving and parityviolating part of the amplitude, respectively. The factor $\Delta m \equiv m_{i}-m_{f}$ multiplying $B$ appears by convention: it is introduced in order to reproduce the parity-violating form factor in the conventional relativistic formalism (see Eq. A7) of the appendix). The factor plays a crucial role in the discussion of Hara's theorem.

Hara's theorem concerns the parityviolating amplitude in the limit of $U$-spin symmetry. ( $U$-spin transformations interchange an $s$ and $d$ quark.) Assuming $U$-spin symmetry Hara's theorem can be easily obtained from Eq. (2). If we have $U$-spin symmetry the mass difference between $p$ and $\Sigma$ vanishes:

$$
m_{\Sigma^{+}}-m_{p}=0
$$


If we also assume that the parity-violating form factor $B$ has no pole in $m_{\Sigma^{+}}-m_{p}$, we find from Eq. (2) that the parity violating amplitude for $\Sigma^{+} \rightarrow p+\gamma$ vanishes. However, as we will see in the following, the assumption that $B$ is non-singular may not be correct in the framework of ChPT.

There are two possible independent observables in this process. Using Eq. (2) and the photon-polarization sum in the gauge $v \cdot A=0$,

$$
\sum_{\lambda} \epsilon_{\lambda}^{\mu}(k) \epsilon_{\lambda}^{\nu}(k)=-g^{\mu \nu}-\frac{k^{\mu} k^{\nu}}{(v \cdot k)^{2}}+\frac{k^{\mu} v^{\nu}+k^{\nu} v^{\mu}}{v \cdot k}, \quad \begin{array}{ccc}
\text { matrix } \\
(4)
\end{array} \quad H=\left(\begin{array}{ccc}
\frac{1}{\sqrt{6}} \Lambda+\frac{1}{\sqrt{2}} \Sigma^{0} & \Sigma^{+} & p \\
\Sigma^{-} & \frac{1}{\sqrt{6}} \Lambda-\frac{1}{\sqrt{2}} \Sigma^{0} & n \\
\Xi^{-} & \Xi^{0} & -\frac{2}{\sqrt{6}} \Lambda
\end{array}\right),
$$

we find for the decay rate the usual expression

$$
\Gamma=\frac{\omega^{3}}{\pi}\left(|A|^{2}+|B|^{2}\right)
$$

where $\omega$ is the photon energy in the labframe given by

$$
\omega=\frac{m_{i}^{2}-m_{f}^{2}}{2 m_{i}} .
$$

As required the decay rates are regular in the chiral limit even if the form factors are singular, since the aforementioned potential singular behavior of $A$ and $B$ is compensated by the phase-space factor $\omega$. The second observable, related to the angular dependence, is the asymmetry parameter given by

$$
\alpha=\frac{2 \Re\left(A B^{*}\right)}{|A|^{2}+|B|^{2}} .
$$

The present data on the decay rates and asymmetry parameters is summarized in Table If.

\section{LEADING ORDER AMPLITUDE}

\section{A. Lagrangian and Feynman diagrams}

We will now turn to the calculation of the hyperon weak radiative decays in leadingorder ChPT in the heavy-baryon formalism. The necessary weak ChPT Lagrangian, up to terms of order $E$, has been given in Ref. 27]. We shall consider only the $C P$ even part of the Lagrangian. The diagrams contributing to the leading-order amplitude are tree diagrams given in Fig. 2. There are two kinds of diagrams: the direct emission diagrams Fig. 2a, and the baryon polediagrams Fig. 2b. Loop diagrams can be omitted in our calculation, since they give rise to contributions of higher order.

Since the full Lagrangian, including the Lagrangian in the weak-interaction sector, has been given elsewhere, we give here only the terms directly relevant to the hyperon weak radiative decay in leading order. The baryons are represented by the usual $S U(3)$

and, since pions do not enter this tree-level description, we can take for the other fields the expansions

$$
\begin{aligned}
& D^{\mu}=\partial^{\mu}-i e Q A^{\mu}, \quad \Delta^{\mu}=0, \quad \sigma=\chi \\
& \rho=0, \quad \lambda=\lambda_{6}, \quad \lambda^{\prime}=\lambda_{7},
\end{aligned}
$$

where $Q$ is the quark charge-matrix

$$
Q=\frac{1}{3} \operatorname{diag}(2,-1,-1),
$$

$\lambda_{6,7}$ are the Gell-Mann matrices (giving rise to $|\Delta S|=1$ transitions),

$$
\lambda_{6}=\left(\begin{array}{ccc}
0 & 0 & 0 \\
0 & 0 & 1 \\
0 & 1 & 0
\end{array}\right), \lambda_{7}=\left(\begin{array}{ccc}
0 & 0 & 0 \\
0 & 0 & -i \\
0 & i & 0
\end{array}\right) \text {, }
$$

and $\chi$ the $S U(3)$-breaking mass matrix

$$
m_{s} \operatorname{diag}(0,0,1) \text {. }
$$

In leading order, the the decuplet does not play a role and we can restrict ourselves to the terms

$$
\begin{aligned}
\mathcal{L}_{s}^{(1,0)}= & i \operatorname{Tr}[\bar{H}[v \cdot D, H]], \\
\mathcal{L}_{s}^{(0,1)}= & A_{1} \operatorname{Tr}[\bar{H}\{\sigma, H\}]+A_{2} \operatorname{Tr}[\bar{H}[\sigma, H]] \\
& +A_{3} \operatorname{Tr}[\bar{H} H] \times \operatorname{Tr}[\sigma], \\
\mathcal{L}_{s}^{(2,0)}= & B_{1} \operatorname{Tr}\left[\bar{H}\left[D^{\mu},\left[D_{\mu}, H\right]\right]\right] \\
& +B_{3} \operatorname{Tr}\left[\bar{H}\left[S_{v}^{\mu}, S_{v}^{\nu}\right]\left\{\left[D_{\mu}, D_{\nu}\right], H\right\}\right] \\
& +B_{4} \operatorname{Tr}\left[\bar{H}\left[S_{v}^{\mu}, S_{v}^{\nu}\right]\left[\left[D_{\mu}, D_{\nu}\right], H\right]\right],
\end{aligned}
$$




$$
\begin{aligned}
\mathcal{L}_{w}^{(0,0)}= & h_{D} \operatorname{Tr}[\bar{H}\{\lambda, H\}]+h_{F} \operatorname{Tr}[\bar{H}[\lambda, H]], \\
\mathcal{L}_{w}^{(1,0)}= & i a_{5} \operatorname{Tr}\left[\bar{H} S_{v}^{\mu}\left\{\lambda,\left[D_{\mu}, H\right]\right\}+\bar{H} S_{v}^{\mu}\left[D_{\mu},\{\lambda,\right.\right. \\
& +i a_{6} \operatorname{Tr}\left[\bar{H} S_{v}^{\mu}\left[\lambda,\left[D_{\mu}, H\right]\right]+\bar{H} S_{v}^{\mu}\left[D_{\mu},\right.\right. \\
& +a_{7} \operatorname{Tr}\left[\bar{H} S_{v}^{\mu}\left\{\left[D_{\mu}, \lambda^{\prime}\right], H\right\}\right] \\
& +a_{8} \operatorname{Tr}\left[\bar{H} S_{v}^{\mu}\left[\left[D_{\mu}, \lambda^{\prime}\right], H\right]\right] .
\end{aligned}
$$

0 thingterms with $a_{7}$ and $a_{8}$ do not contribute to hyperon weak radiative decays in leading oftder, and can be ignored in the following. The diagrams in Fig. 2 lead to the fol, [ factors A in leading order ChPT,

$$
\begin{gathered}
A_{\Lambda(1 \rightarrow T)^{\gamma}}=-\frac{e B_{3}}{3 \sqrt{6}}\left(\frac{3 h_{F}+h_{D}}{m_{\Lambda}-m_{N}}-3 \frac{h_{F}-h_{D}}{m_{\Sigma}-m_{N}}\right), \\
A_{\Sigma^{+} \rightarrow p \gamma}=0, \quad(18 \mathrm{a}) \\
A_{\Sigma^{0} \rightarrow n \gamma}=\sqrt{3} A_{\Lambda \rightarrow n \gamma}, \\
A_{\Xi^{0} \rightarrow \Lambda \gamma}=-\frac{e B_{3}}{3 \sqrt{6}}\left(\frac{3 h_{F}-h_{D}}{m_{\Xi}-m_{\Lambda}}-3 \frac{h_{F}+h_{D}}{m_{\Xi}-m_{\Sigma}}\right) \\
=-\frac{m_{\Lambda}-m_{N}}{m_{\Xi}-m_{\Lambda}} \frac{m_{\Sigma}-m_{N}}{m_{\Xi}-m_{\Sigma}} A_{\Lambda \rightarrow n \gamma}, \quad(18 \mathrm{~d}) \\
A_{\Xi^{0} \rightarrow \Sigma^{0} \gamma}=\sqrt{3} A_{\Xi^{0} \rightarrow \Lambda \gamma} \\
=-\sqrt{3} \frac{m_{\Lambda}-m_{N}}{m_{\Xi}-m_{\Lambda}} \frac{m_{\Sigma}-m_{N}}{m_{\Xi}-m_{\Sigma}} A_{\Lambda \rightarrow n \gamma},
\end{gathered}
$$$$
\text { Eq. (17), were not taken into account in pre- }
$$$$
\text { vious ChPT calculations. }
$$

The parameters $A_{1}, A_{2}$ and $A_{3}$ in the strong Lagrangian $\mathcal{L}_{s}^{(0,1)}$ determine the four $S U(2)$ invariant masses of the octet baryons up to first order in $m_{s}$. Therefore, they provide one prediction, which is the Gell-MannOkubo mass relation, and fit the physical baryon masses within about $5 \%$. We will choose these parameters such that the baryon masses are fitted best. Except for these mass terms in the strong Lagrangian $\mathcal{L}_{s}^{(0,1)}$, all the terms in the Lagrangian obey $S U(3)$ symmetry. Therefore, all $S U(3)$ breaking effects in the amplitudes in our formulation are due to the intermediate baryon propagator in the pole-diagrams in Fig. (2b). Since we have chosen $m_{u}=m_{d}=0$, and the small mass effects due to the electromagnetic interaction can be ignored here, the baryon masses obey isospin symmetry and we will use the obvious notation $m_{N}$, $m_{\Lambda}, m_{\Sigma}$, and $m_{\Xi}$ to represent the average mass of the corresponding isospin multiplets. Note that in calculating the decay rates the phase space gives rise to additional sources of $S U(3)$ breaking mass differences. However, in that case, we shall use the observed masses.

The terms with $B_{1}, \ldots, B_{3}$ in the stronginteraction Lagrangian $\mathcal{L}_{s}^{(2,0)}$ enter the amplitude for weak radiative decays through the baryon electromagnetic vertex in the pole-diagrams of Fig. (2b), while $h_{D}$ and $h_{F}$ in the weak-interaction Lagrangian $\mathcal{L}_{w}^{(0,0)}$ enter through the weak baryon-mixing in these same diagrams.

Finally, the terms containing the parameters $a_{5}, \ldots, a_{8}$ in the weak Lagrangian $\mathcal{L}_{w}^{(1,0)}$ give rise to the direct emission diagrams shown in Fig. (2a). However, since $\left[\lambda_{7}, Q\right]=$ and

$$
A_{\Xi^{-} \rightarrow \Sigma^{-} \gamma}=0 .
$$

The final result in Eq. (18d) follows from the Gell-Mann-Okubo mass relation which is, as discussed above, satisfied to the order we consider. The expressions for the parityconserving amplitude was also be obtained [8] from arguments based on the $U$-spin symmetry (which is satisfied in this order due to the structure of the quark charge-matrix $Q$ in Eq. (10)) of the magnetic moments.

For the parity-violating amplitude $B$ we find,

$$
\begin{gathered}
B_{\Lambda \rightarrow n \gamma}=0, \\
B_{\Sigma^{+} \rightarrow p \gamma}=\frac{e\left(a_{5}-a_{6}\right)}{m_{\Sigma}-m_{N}}, \\
B_{\Sigma^{0} \rightarrow n \gamma}=0, \\
B_{\Xi^{0} \rightarrow \Lambda \gamma}=0, \\
B_{\Xi^{0} \rightarrow \Sigma^{0} \gamma}=0,
\end{gathered}
$$


and

$$
B_{\Xi^{-} \rightarrow \Sigma^{-} \gamma}=-\frac{e\left(a_{5}+a_{6}\right)}{m_{\Xi}-m_{\Sigma}} .
$$

Note that the mass differences, $\Delta m$, in the denominators in Eq. (19) arise because of the $\Delta m$ in the parity-violating part of the general amplitude Eq. (2). From the point of view of ChPT, $a_{i}$ are the fundamental parameters that should be treated as constants, i.e., they can not compensate for the $\Delta m$ in the denominators in Eq. (19). As a result, the $B$ form factors become singular in the $S U(3)$ invariant limit, contrary to the usual implicit assumption in the derivation of Hara's theorem.

Let us now take a closer look at our results. The pole-diagrams only contribute to the parity-conserving form factor $\mathrm{A}$, in accordance with the Lee-Swift theorem [31]. Since the pole contributions to the charged decay modes $\Sigma^{+} \rightarrow p+\gamma$ and $\Xi^{-} \rightarrow \Sigma^{-}+\gamma$ cancel, we find a nonzero parity-conserving form factor only for the neutral decays $\Lambda \rightarrow$ $n+\gamma, \Sigma^{0} \rightarrow n+\gamma, \Xi^{0} \rightarrow \Lambda+\gamma$, and $\Xi^{0} \rightarrow \Sigma^{0}+\gamma$.

The terms in weak Lagrangian in Eq. (17) all contain $[Q, H]$. Since $[Q, H]=0$ for neutral baryons, the direct emission diagrams do not contribute to any of the neutral decays. For the same reason also the parameters $B_{1}$ and $B_{4}$ do not give a contributions to the neutral decays in the pole diagrams.

Since for all the decays either $A$ or $B$ vanishes we immediately conclude that the asymmetry parameters, defined by Eq. (7), still vanishes in this (leading) order. However, we can show, by qualitative arguments, that this does not need to imply a contradiction between ChPT and the measured asymmetry parameters in Table II. Assume, in the spirit of ChPT, that the charged decay modes can be expanded as

$$
A=a_{1} \lambda ; \quad B=b_{0}+\lambda b_{1},
$$

with $\lambda \approx 0.3\left(\approx m_{s} / \Lambda_{\chi}\right)$, and that $a_{1}, b_{0}$, and $b_{1}$ are of about equal magnitude. It leads, using Eq. (7), to an asymmetry parameter of about 0.6 in magnitude, which is roughly in agreement with the data.

In leading order, we find from Eq. (18)

$$
\begin{gathered}
\mathcal{M}_{\Sigma^{0} \rightarrow n+\gamma}^{\mu}=\sqrt{3} \mathcal{M}_{\Lambda \rightarrow n+\gamma}^{\mu} \\
\mathcal{M}_{\Xi^{0} \rightarrow \Lambda+\gamma}^{\mu}=-\frac{1}{\sqrt{3}} \frac{m_{\Lambda}-m_{N}}{m_{\Xi}-m_{\Lambda}} \frac{m_{\Sigma}-m_{N}}{m_{\Xi}-m_{\Sigma}} \mathcal{M}_{\Sigma^{0} \rightarrow n+\gamma}^{\mu}
\end{gathered}
$$

$$
\mathcal{M}_{\Xi^{0} \rightarrow \Sigma^{0}+\gamma}^{\mu}=\sqrt{3} \mathcal{M}_{\Xi^{0} \rightarrow \Lambda+\gamma}^{\mu} .
$$

Therefore, all ratios of the neutral decay amplitudes depend only on the baryon masses and not on any constant from $\mathcal{L}_{s}^{(2,0)}, \mathcal{L}_{w}^{(0,0)}$ or $\mathcal{L}_{w}^{(1,0)}$. Their magnitudes, on the other hand, also depend on a particular combination of the three parameters $B_{3}, h_{D}, h_{F}$.

\section{INCLUSION OF THE 27-PLET}

The Lagrangian Eq. (17) corresponds to the part of the weak interaction that transforms as $\left(8_{L}, 1_{R}\right)$ under $S U(3)_{L} \times S U(3)_{R}$. Since the weak interaction consists of a product of two left-handed flavor-SU(3) currents, the effective chiral Lagrangian also has a $\left(27_{L}, 1_{R}\right)$ component. This 27 -plet part of the weak Lagrangian can be included in the same way as the octet part [27]. Closer inspection shows that inclusion of the 27-plet corresponds simply to replacing $h_{D}$ and $h_{F}$ in Eqs. (18) and (19) by

$$
h_{D} \rightarrow h_{D}+2 h_{27}
$$

in the charged channels, and by

$$
h_{D} \rightarrow h_{D}-3 h_{27}
$$

in the neutral channels, where $h_{27}$ is the coupling constant from the leading-order weak interaction Lagrangian that transforms as a 27 -plet. From the $\Delta I=1 / 2$ rule of the weak nonleptonic decays, $h_{27}$ is expected to be a small parameter compared to $h_{D}$ and $h_{F}$. While it enters the charged and neutral channels differently, the inclusion of the 27plet into the analysis does not alter our results, both for the decay rates and the asymmetry parameters.

\section{DISCUSSION OF THE RESULTS}

Before discussing the relations Eq. (23) we first investigate closer the different parameters in Eqs. (18) and (19) which contribute to the amplitude. Since we find a distinct results for the neutral and charged channels, we will discuss them separately. 


\section{A. Charged channels and Hara's theorem}

Relevant for the two charged channels are the two parameters $a_{5}$ and $a_{6}$ from the next-to-leading order weak Lagrangian $\mathcal{L}_{w}^{(1,0)}$. Clearly, we can fit these two parameters to the two experimental decay rates as given in Table 1 . Due to the quadratic relation between the amplitude and the decay rate we find the following four combinations for the (dimensionless) parameters $a_{5}$ and $a_{6}$ :

$$
a_{5}=2.8 \epsilon \times 10^{-8} \text { and } a_{6}=-1.6 \epsilon \times 10^{-8} \text {, }
$$

or

$$
a_{5}=-1.6 \epsilon \times 10^{-8} \text { and } a_{6}=2.8 \epsilon \times 10^{-8},
$$

where $\epsilon= \pm 1$. With these parameters the experimental data is reproduced. However, no prediction can be made yet.

More interestingly, due to the direct emission diagrams from $\mathcal{L}_{w}^{(1,0)}$, Hara's theorem is violated: Even in the $U$-spin symmetric limit, the parity-violating part of the decay amplitude is nonzero.

\section{B. Neutral channels}

As can be seen in Eq. (18) the relevant parameters for the neutral channels are $B_{3}$, $h_{D}$, and $h_{F}$. The parameter $B_{3}$ is from the strong interaction Lagrangian Eq. (15), and determines (together with the constant $B_{4}$ ) the magnetic moments of the octet baryons. The first column of Table [1] shows the magnetic moments of the baryons in leading order ChPT. Fitting $B_{3}$ to the experimental data, shown in the second column of Table II. gives

$$
B_{3}=-1.3 \mathrm{GeV}^{-1} .
$$

The resulting fitted magnetic moments as given in the third column of Table III. Note that these results give rise to the Coleman and Glashow [29] relations between the baryon magnetic moments.

The parameters $h_{D}$ and $h_{F}$ can be obtained from hyperon nonleptonic decay.
Considering $s$-wave nonleptonic decay data gives the best values [30],

$$
h_{D}=-0.58 \mu ; h_{F}=1.40 \mu,
$$

where $\mu=G_{F} m_{\pi}^{2} \sqrt{2} f_{\pi} \approx 3 \times 10^{-8} \mathrm{GeV}$.

Using these values, together with that for $B_{3}$, we arrive at the decay rates for hyperon radiative decay as given in Table III. It shows a huge disagreement with the observed rates. The difference between the experimental and predicted decay rate is more than a factor of 200 for $\Lambda \rightarrow n+\gamma$, while the difference for the other channels is about two orders of magnitude.

This discrepancy, however, is highly dependent on the values used for $h_{D}$ and $h_{F}$. Independent of any particular values chosen for the parameters from the Lagrangian we can use Eq. (23) to predict three ratios between the four neutral decay rates. We find

$$
\begin{gathered}
\frac{\Gamma_{\Sigma^{0} \rightarrow n+\gamma}}{\Gamma_{\Lambda \rightarrow n+\gamma}}=8.1, \\
\frac{\Gamma_{\Xi^{0} \rightarrow \Lambda+\gamma}}{\Gamma_{\Lambda \rightarrow n+\gamma}}=4.9(0.59 \pm 0.14), \\
\frac{\Gamma_{\Xi^{0} \rightarrow \Sigma^{0}+\gamma}}{\Gamma_{\Lambda \rightarrow n+\gamma}}=3.7(1.99 \pm 0.42),
\end{gathered}
$$

where the experimental values obtained from Table 1 are written between parentheses. While the first ratio can not be obtained from experimental data, since $\Sigma^{0} \rightarrow n+\gamma$ has not been measured, the predicted values for the other ratios is are only within about a factor 8 and 2 in accordance with the observed ratios. In contrast to the case of the asymmetry parameters one can not hope that this disagreement may be resolved by higher order effects, if ChPT is a consistent expansion scheme with the next-toleading order corrections about $30 \%$ suppressed.

\section{SUMMARY AND CONCLUSIONS}

We have studied the process of hyperon weak radiative decay in the framework of heavy baryon chiral perturbation theory [25]. In particular we have put emphasis on the effect of including a complete next-toleading order weak Lagrangian [27] in the 
description. We used it to obtain the leading order decay amplitudes. In previous calculations of hyperon weak radiative decays these contributions to the amplitudes have been ignored.

In the leading order, all the ratios for decay amplitudes of the four neutral channels, $\Lambda \rightarrow n+\gamma, \Sigma^{0} \rightarrow n+\gamma, \Xi^{0} \rightarrow$ $\Sigma^{0}+\gamma$, and $\Xi^{0} \rightarrow \Lambda+\gamma$, are simple functions of the baryon masses only. This follows from the observation that, for the neutral channels, the direct-emission contribution vanishes and only pole-diagrams contribute. Compared with experiment these ratios are up to a factor 8 off. It is interesting to note that taking the values of $h_{D}$ and $h_{F}$ from analysis of nonleptonic weak decays of hyperons leads to predictions which disagree with the observed decay rates for more than two orders of magnitude.

Clearly, these disagreements indicate something deficient about the applications of ChPT to the neutral decays. One possible solution to this problem is to assume that at leading order, the weak Lagrangian consists of additional terms, in particular terms that are not invariant under $U$-spin transformations, or $C P S$, for some reason. Alternatively, it may indicate that resonances, such as the vector mesons, have to included in the analysis of ChPT as hinted by the relative success of the vector-meson-dominance models 16.

For the two charged channels $\Sigma^{+} \rightarrow p+\gamma$ and $\Xi^{-} \rightarrow \Sigma^{-}+\gamma$ no such relations can be extract from ChPT to this order. Contrary to the case of the neutral channels, only the direct emission diagrams contribute. The contributions of all the pole-diagrams totally cancel each other at this order. The two observed decay rates can be used to fix exactly the two parameters $a_{5}$ and $a_{6}$ from the next-to-leading order weak Lagrangian. Although we derive no prediction within the charged channels, the parameters extracted will be relevant for other observables such as weak semi-leptonic decays, making a future comparison feasible.

We have shown that, even in leading order ChPT, Hara's theorem is violated by direct emission diagrams contributing to the parity-violating part of the amplitude. Going through the original assumptions in the proof of the theorem, our result indicates that the parity-violating form factors in the amplitude are singular in the limit of $U$ - spin (or $S U(3)$ ) symmetry in the context of ChPT. This singular behavior leads to the failure of Hara's theorem.

The asymmetry parameters vanish in our leading-order calculation. However, it can still potentially be accounted for by the higher order effect within the context of ChPT. In other words, our results indicate that the asymmetry parameters are sensitive to loop effects and parameters in the higherorder Lagrangian.

\section{ACKNOWLEDGMENTS}

This work was supported by the National Science Council of the R.O.C. under Contracts Nos. NSC85-2112-M-007-032, NSC85-2112-M-007-029, and NSC85-2112M-007-0.

\section{APPENDIX: GENERAL FORM OF WEAK RADIATIVE DECAY IN HEAVY-BARYON CHIRAL PERTURBATION THEORY}

In this appendix we briefly review the heavy-baryon formulation of ChPT, and give the general amplitude of weak radiative decay in this formulation.

The nucleon mass in the chiral limit, $\dot{m}$, is comparable with the chiral symmetry breaking scale $\Lambda_{\chi}$. To make a consistent chiral expansion possible it can be removed by redefining the baryon field according to 25

$$
B_{v}=\mathrm{e}^{i \dot{m} v \cdot x} B
$$

where $v^{\mu}$ is the baryon four-velocity satisfying $v^{2}=1$. Next, one defines the projected fields

$$
H=P_{v}^{+} B_{v} ; h=P_{v}^{-} B_{v},
$$

where $P_{v}^{+}$and $P_{v}^{-}$are the projection operators

$$
P_{v}^{ \pm}=\frac{1 \pm \psi}{2}
$$

The minus component field $h$ is suppressed by $1 / \dot{m}$ compared to $H$. It can be easily seen that, in momentum space, derivatives of $H$ produce powers of

$$
k^{\mu}=p^{\mu}-\dot{m} v^{\mu},
$$


with $p^{\mu}$ the four-momentum of the baryon, which is (for processes at low energies) a small quantitiy. This residual baryon momentum, $k$, is the effective expansion parameter in this formulation of baryon ChPT. Effects of $1 / \dot{m}$ can arise through $h$ in higher order. However, these $1 / \dot{m}$ corrections can be absorbed in the higher-order counterterms of the theory [27.

The baryon field $H$ satisfies the Dirac equation

$$
(v \cdot k-\bar{m}) H=0
$$

where $\bar{m} \equiv m_{B}-\dot{m}$ is defined as the residual mass of the baryon.

The general amplitude for the weak radiative decay

$$
B(p) \rightarrow B^{\prime}\left(p^{\prime}\right)+\gamma
$$

is given by

$$
\begin{aligned}
& \epsilon_{\mu}(q) \mathcal{M}^{\mu}= \\
& \quad \epsilon_{\mu}(q) \bar{u}\left(p^{\prime}\right) i \sigma^{\mu \nu} q_{\nu}\left(A+B \gamma_{5}\right) u(p) .
\end{aligned}
$$

Defining the operator $S_{v}^{\mu} \equiv(1 / 2) P_{v}^{+} \gamma^{\mu} \gamma_{5} P_{v}^{+}$and using

$$
\begin{gathered}
P_{v}^{+} \gamma_{5} P_{v}^{+}=0, P_{v}^{+} \gamma^{\mu} P_{v}^{+}=P_{v}^{+} v^{\mu} \\
P_{v}^{+} \sigma^{\mu \nu} P_{v}^{+}=-2 i\left[S_{v}^{\mu}, S_{v}^{\nu}\right] \\
P_{v}^{+} \sigma^{\mu \nu} \gamma_{5} P_{v}^{+}=-2 i\left(v^{\mu} S_{v}^{\nu}-v^{\nu} S_{v}^{\mu}\right)
\end{gathered}
$$

and Eq. (A5), we find that the general form of weak radiative decay in the heavy baryon formalism is given by

$$
\begin{aligned}
& \epsilon_{\mu}(q) \mathcal{M}^{\mu}= \\
& \quad 2 \epsilon_{\mu}(q) \bar{U}_{v}\left(k^{\prime}\right)\left(q_{\nu}\left[S_{v}^{\mu}, S_{v}^{\nu}\right] A\right. \\
& \left.\quad+\left(S_{v}^{\mu} \Delta m_{B}-v^{\mu} S_{v} \cdot q\right) B\right) U_{v}(k),
\end{aligned}
$$

where $\Delta m_{B}$ is the mass difference between the initial and final baryon. In the gauge $v \cdot A=0$ we then finally arrive at the form as is used in the main text Eq. (2).

[1] K. Larsen et al., Phys. Rev. D47, 799 (1993); S. F. Biagi et al., Z. Phys. C30,
201 (1986); B. L. Roberts et al., Nucl. Phys. A479, 75 (1988); B. L. Roberts et al., Nucl. Phys. (Proc. Suppl.) B13, 449 (1990); A. J. Noble et al., Phys. Rev. Lett. 69, 414 (1992).

[2] C. James et al., Phys. Rev. Lett. 64, 843 (1990); N. Yeh et al., Phys. Rev. D10, 3545 (1974).

[3] S. Teige et al., Phys. Rev. Lett. 63, 2717 (1989); J. R. Bensinger et al., Phys. Lett. B215, 195 (1988).

[4] S. Timm et al., Phys. Rev. D51, 4638 (1995).

[5] M. Foucher et al., Phys. Rev. Lett. 68, 3004 (1992); L. K. Gershwin et al., Phys. Rev. 188, 2077 (1969); N. P. Hessey et al., Z. Phys. C42, 175 (1989); M. Kobayashi et al., Phys. Rev. Lett. 59, 868 (1987); S. F. Biagi et al., Z. Phys. C28, 495 (1985); A. Manz et al., Phys. Lett. B96, 217 (1980).

[6] T. Dubbs et al., Phys. Rev. Lett. 72, 808 (1994); S. F. Biagi et al., Z. Phys. C35, 143 (1987).

[7] Y. Hara, Phys. Rev. Lett. 12, 378 (1964); S. Y. Lo, Nuovo Cimento 37, 753 (1965); K. Tanaka, Phys. Rev. 140, B463 (1965); M. Gourdin, Unitary symmetry (NorthHolland, Amsterdam, 1967).

[8] M. K. Gaillard, Nuovo Cimento A6, 559 (1971).

[9] V. I. Zakharov and A. B. Kaidalov, Sov. J. Nucl. Phys. 5, 259 (1967); G. R. Farrar, Phys. Rev. D4, 212 (1971); J. H. Reid and N. N. Trofimenkoff, Nucl. Phys. B82, 397 (1974).

[10] J. Pati, Phys. Rev. 130, 2097 (1963); R. H. Graham and S. Pakvasa, Phys. Rev. 140, B1144 (1965); M. D. Scadron and L. Thebaud, Phys. Rev. D8, 2190 (1973); L. Copley et al., Nucl. Phys. B13, 303 (1969); B. R. Holstein, Nuovo Cimento 2A, 561 (1971); Y. I. Skovpen, Sov. J. Nucl. Phys. 34, 773 (1981); M. D. Scadron and M. Visinescu, Phys. Rev. D28, 1117 (1983); L. F. Li and Y. Liu, Phys. Lett. B195, 281 (1987); M. K. Gaillard, Phys. Lett. B211, 189 (1988); G. Nardulli, Nuovo Cimento A100, 485 (1988); Y. Liu, Z. Phys. C45, 345 (1989); R. E. Karlson and M. D. Scadron, Z. Phys. C52, 325 (1991); A. Maity and P. Mahato, Nuovo Cimento A104, 269 (1991).

[11] Y. I. Kogan and M. A. Shifman, Sov. J. Nucl. Phys. 38, 628 (1983).

[12] F. E. Close and H. R. Rubinstein, Nucl. Phys. B173, 477 (1980); I. Picek, Phys. Rev. D21, 3169 (1980); M. B. Gavela et al., Phys. Lett. B101, 417 (1981); K. Rauh, 
Z. Phys. C10, 81 (1981); D. Palle, Phys. Rev. D36, 2863 (1987); P. Singer, Phys. Rev. D42, 3255 (1990); R. E. Karlsen, W. H. Ryan, and M. D. Scadron, Phys. Rev. D43, 157 (1991).

[13] N. Vasanti, Phys. Rev. D13, 1889 (1976).

[14] A. N. Kamal and Riazuddin, Phys. Rev. D28, 2317 (1983).

[15] M. A. Ahmed and C. G. Ross, Phys. Lett. B59, 293 (1975); F. J. Gilman and M. B. Wise, Phys. Rev. D19, 976 (1979); A. N. Kamal and R. C. Verma, Phys. Rev. D26, 190 (1982); C. H. Lo, Phys. Rev. D26, 199 (1982); S.G. Kamath, Nucl. Phys. B198, 61 (1982); J. O. Eeg, Z. Phys. C21, 253 (1984); M. K. Gaillard, X. Q. li and S. Rudaz, Phys. Lett. B158, 158 (1985); L. Bergström and P. Singer, Phys. Lett. B169, 297 (1986); Z. Phys. C37, 281 (1988); R. Safadi and P. Singer, Phys. Rev. D37, 697 (1988); R. Verma and A. Sharma, Phys. Rev. D38, 1443 (1988); T. Uppal and R. Verma, Z. Phys. C52, 307 (1991).

[16] K. Gavroglu and H. P. W. Gottlieb, Nucl. Phys. B79, 168 (1974); P. Żenczykowski, Phys. Rev. D40, 2290 (1989); D44, 1485 (1991); D50, 3285 (1994).

[17] V. M. Khatsymovski, Sov. J. Nucl. Phys. 45, 116 (1987); 46, 768(E) (1987); 46, 496 (1987); I. I. Balitsky, V. M. Braun, and A. V. Kolesnichenko, Sov. J. Nucl. Phys. 44, 1028 (1986); Nucl. Phys. B312, 509 (1989); C. Goldman and C. O. Escobar, Phys. Rev. D40, 106 (1989).

[18] M. A. Shifman, A. I. Vainshtein, and V. I. Zakharov, Phys. Rev. D18, 2583 (1978); A. Cohen, Phys. Lett. B160, 177 (1985); W. F. Kao and H. J. Schnitzer, Phys. Rev. D37, 1912 (1988); Phys. Lett. B183, 361 (1987); R. W. Brown and E. A. Paschos, Nucl. Phys. B319, 623 (1989); P. Asthana and A. Kamal, Few Body Systems 11, 1 (1991).

[19] H. Neufeld, Nucl. Phys. B402, 166 (1993).

[20] E. Jenkins, M. Luke, A. V. Manohar, and M. J. Savage, Nucl. Phys. B397, 84 (1993).

[21] J. Lach and P. Żenczykowski, Int. J. Mod. Phys. A10, 3817 (1995).

[22] B. Bassalleck, Nucl. Phys. A547, 299 (1992).

[23] S. Weinberg, Phys. Rev. Lett. 18, 188 (1967); J. Schwinger, Phys. Lett. B24, 473 (1967); R. Dashen, Phys. Rev. 183, 1245 (1969); R. Dashen and M. Weinstein, Phys. Rev. 183, 1261 (1969); 188, 2330 (1969); L. F. Li and H. Pagels, Phys. Rev. Lett. 26, 1204 (1971); 27, 1089 (1971); Phys. Rev. D5, 1509 (1972); P. Lan- gacker and H. Pagels, Phys. Rev. D8, 4595 (1973); D10, 2904 (1974); J. Gasser and H. Leutwyler, Phys. Lett. B125, 321 (1983); Ann. Phys. 158, 142 (1984); Nucl. Phys. B250 (1985) 465, 517, 539. A. V. Manohar and H. Georgi, Nucl. Phys. B234, 189 (1984); J. Bijnens, H. Sonoda, and M. Wise, Nucl. Phys. B261, 185 (1985); J. Gasser, M. Sainio, and A. S̆varc, Nucl. Phys. B307, 779 (1988); A. Krause, Helv. Phys. Acta 63, 3 (1990).

[24] S. Weinberg, Physica 96A, 327 (1979).

[25] E. Jenkins and A. V. Manohar, Phys. Lett. B255, 558 (1991).

[26] S. Weinberg, Nucl. Phys. B363 3 (1991).

[27] J. W. Bos, D. Chang, S. C. Lee, Y. C. Lin, and H. H. Shih, Phys. Rev. D51, 6308 (1995).

[28] J. F. Donoghue, E. Golowich, and B. R. Holstein, Dynamics of the standard model (Cambridge, New York, 1992).

[29] S. Coleman and S. L. Glashow, Phys. Rev. Lett. 6, 423 (1961).

[30] E. Jenkins, Nucl. Phys. B375, 561 (1992).

[31] B. W. Lee and A. R. Swift, Phys. Rev. 136B, 228 (1964).

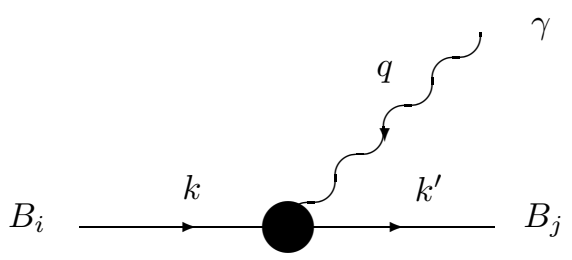

FIG. 1. Kinematics for weak radiative hyperon decays. $B_{i}$ and $B_{f}$ denote the initial and final hyperon, respectively. The baryon momenta $k$ and $k^{\prime}$ are residual momenta, defined in the appendix. 


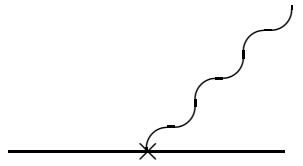

(a)
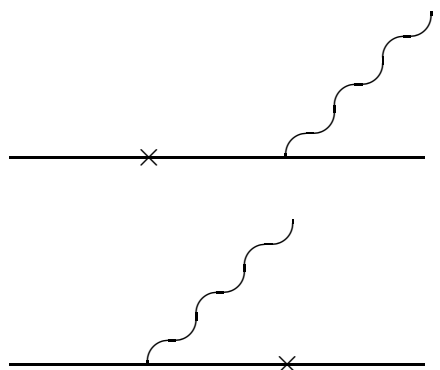

(b)

FIG. 2. Feynman diagrams for weak radiative hyperon decay in leading-order chiral perturbation theory. The cross-sign denotes the weak interaction.

TABLE I. Present status of decay rates and asymmetry parameters. The numbers are the combined weighted mean from Ref. 21. Both the decay rate and the asymmetry parameter for $\Sigma^{0} \rightarrow \Lambda+\gamma$ have not been measured.

\begin{tabular}{|c|c|c|c|}
\hline$B_{i} \rightarrow B_{f}+$ & $\Gamma\left[10^{-18} \mathrm{GeV}\right]$ & $\alpha$ & \\
\hline$\Lambda \rightarrow n+\gamma$ & $4.07 \pm 0.35$ & & \\
\hline & 36 & $0.43 \pm$ & \\
\hline- & 8 & $0.20 \pm$ & \\
\hline$\Sigma^{+}$ & 10 & -0 . & \\
\hline$\Xi^{-} \rightarrow \Sigma^{-}+\gamma$ & $0.51 \pm 0.092$ & $1.0 \pm 1.3$ & \\
\hline
\end{tabular}

TABLE II. Magnetic moments of the octet baryons and the transitional moment $\Sigma^{0} \rightarrow \Lambda+\gamma$ in leading-order chiral perturbation theory. The first column contains the expressions in leading-order ChPT, the second column the experimental values, and the third column the fitted values, with $B_{3}=-1.13 \mathrm{GeV}^{-1}$ and $B_{4}=-0.82 \mathrm{GeV}^{-1}$. The average difference between the fitted and experimental moments is $19 \%$. The constants $B_{3}$ and $B_{4}$ are from the next-to-leading order strong Lagrangian $\mathcal{L}_{s}^{(2,0)}$ (see Eq. (15)). Note that only the constant $B_{3}$ plays a role in hyperon radiative decays.

\begin{tabular}{cccc}
\hline \hline$B$ & $\mathcal{M}_{\text {th }}[e]$ & $\mathcal{M}_{\exp }\left[\mu_{N}\right]$ & $\mathcal{M}_{\text {fitted }}\left[\mu_{N}\right]$ \\
\hline$p$ & $-B_{3} / 3-B_{4}$ & 2.79 & 2.25 \\
$n$ & $2 B_{3} / 3$ & -1.91 & -1.41 \\
$\Lambda$ & $B_{3} / 3$ & -0.61 & -0.71 \\
$\Sigma^{+}$ & $-B_{3} / 3-B_{4}$ & 2.42 & 2.25 \\
$\Sigma^{-}$ & $-B_{3} / 3+B_{4}$ & -1.16 & -0.84 \\
$\Xi^{-}$ & $-B_{3} / 3+B_{4}$ & -0.68 & -0.84 \\
$\Xi^{0}$ & $2 B_{3} / 3$ & -1.25 & -1.41 \\
$\Sigma^{0} \rightarrow \Lambda$ & $B_{3} / \sqrt{3}$ & \pm 1.6 & - \\
\hline \hline
\end{tabular}

TABLE III. Decay rates for the four neutral hyperon radiative decays taking for the parameters $h_{D}$ and $h_{F}$ the values in Eq. (29) obtained from nonleptonic hyperon decays. The first column shows the observed rates from Ref. [22]. All decay rates are in units of $10^{-18} \mathrm{GeV}$.

\begin{tabular}{ccc}
\hline \hline$B_{i} \rightarrow B_{f}+\gamma$ & $\Gamma_{\exp }$ & $\Gamma_{\text {Eq. }}(29)$ \\
\hline$\Lambda \rightarrow n+\gamma$ & 4.07 & 0.018 \\
$\Sigma^{0} \rightarrow n+\gamma$ & - & 0.16 \\
$\Xi^{0} \rightarrow \Lambda+\gamma$ & 2.4 & 0.087 \\
$\Xi^{0} \rightarrow \Sigma^{0}+\gamma$ & 8.1 & 0.067 \\
\hline \hline
\end{tabular}

